\title{
Análise da Produção de Artigos Científicos sobre o Lazer: Uma Revisão ${ }^{1}$
}

\author{
Maiana Farias Oliveira Nunes ${ }^{2}$ \\ Claudio Simon Hutz \\ Universidade Federal do Rio Grande do Sul
}

\begin{abstract}
RESUMO - Este estudo objetivou analisar artigos científicos sobre a temática "lazer", por meio de uma pesquisa documental. Foi feita uma busca na Biblioteca Virtual de Saúde, havendo uma predominância de estudos da Educação Física, Psicologia, Epidemiologia, Enfermagem e Nutrição. O lazer foi abordado no sentido específico das atividades físicas realizadas durante os momentos de lazer ou dos tipos de atividade de lazer, seguidos por aqueles que abordavam teórica ou empiricamente o conceito genérico de lazer e as atitudes frente o lazer. Verificou-se menor proporção de estudos sobre benefícios psicológicos e sociais relacionados às práticas de lazer. Entre as considerações finais, espera-se que novos estudos sobre a temática ampliem seu escopo, indo além dos benefícios físicos promovidos com a prática de atividades de lazer.
\end{abstract}

Palavras-chave: lazer, ócio, tempo livre, uso do tempo, estado da arte

\section{Analysis of Scientific Papers about Leisure: A Review}

\begin{abstract}
This study aimed to analyze scientific papers on the theme "leisure", by means of a documental research. A search was performed at the "Biblioteca Virtual de Saúde", with a predominance of studies in Psychology, Physical Education, Epidemiology, Nursing and Nutrition. Leisure has been approached in the specific sense of physical activities performed during leisure time, followed by studies describing types of leisure activities, those that addressed theoretically or empirically the generic concept of leisure and attitudes toward leisure activities. There were few studies regarding psychological and social benefits related to leisure-time activities. Among the concluding remarks, we expect that further studies on the subject should broaden their scope, reaching beyond the physical benefits promoted the practice of leisure activities.
\end{abstract}

Keywords: leisure, leisure time, free time, time use, state of art

O lazer é uma temática que vem sendo estudada cientificamente desde o século XIX, sob diferentes perspectivas, a saber, sociológica, econômica, psicológica, e outras. Na perspectiva psicológica, o lazer seria a satisfação de uma necessidade humana complexa, que é colocada em prática por meio de experiências que são individualmente definidas como prazerosas.

Desse modo, cada pessoa define o lazer de acordo com seus gostos e com os recursos disponíveis para satisfazer suas necessidades, e poderá valorizar os resultados de maneira diferenciada, de acordo com seu sistema de valores e aspirações. O caráter de "lazer" da atividade vem do significado que a pessoa dá a uma atividade, e não à atividade em si. Com isso, o lazer pode assumir formas diversas para diferentes pessoas, ou seja, uma mesma atividade pode ser vista como obrigação ou como lazer (Witt \& Bishop, 2009).

Ainda sob a ótica da Psicologia, o lazer pode ser pensado como uma das áreas, fora do contexto do trabalho, em que o indivíduo pode desenvolver seu potencial e realizar suas necessidades de vida. Assim, o lazer pode ser compreendido no sentido do desenvolvimento dos interesses fora do contexto do trabalho, como um dos fatores que pode contribuir para sua qualidade de vida (Armstrong \& Rounds, 2008; Dik \& Hansen, 2008; Gaudron \& Vautier, 2007; Hur, McGue, \& Iacono, 1996). Com outro enfoque, a Psicologia também tem abordado os riscos psicossociais relacionados

1 Apoio: $\mathrm{CNPq}$

2 Endereço para correspondência: Av. Marginal Leste, $n^{\circ} 3600, \mathrm{KM} 132$, Bairro dos Estados, Balneário Camboriú, SC, Brasil, CEP 88339-125. E-mail:maiananunes@mac.com à prática de certas atividades de lazer, tais como o consumo de drogas e a violência, e a reflexão sobre intervenções que estimulem práticas de lazer mais saudáveis entre os jovens (Carvalho \& Carlini-Cotrim, 1992; Pratta \& Santos, 2007; Silva \& Salles, 2010). Mais detalhadamente, o envolvimento em atividades de lazer e o uso que se faz do tempo livre podem trazer consequências positivas ou negativas para as pessoas, de modo que elas podem desenvolver seus potenciais e aumentar seu bem-estar, por um lado, ou usar o tempo de forma alienada, que não contribui para seu desenvolvimento pessoal (Aquino \& Martins, 2007).

Nessa direção, Sarriera, Tatim, Coelho e Bucker (2007) referem a importância da realização de estudos que tratem do tempo livre como uma oportunidade para o desenvolvimento pessoal saudável, no sentido de auxiliar a elaboração de políticas públicas e intervenções que tragam alternativas saudáveis de lazer. Os autores destacam que, em alguns casos, o tempo livre pode ser associado a momentos de tédio e de não saber o que fazer com o tempo.

Nessas situações, o uso do tempo livre poderá assumir o caráter de risco para situações nocivas para a saúde física e psicológica da pessoa. Como uma das soluções possíveis, sugere-se a participação da família, da comunidade e da escola na organização do tempo livre, no sentido de oferecerem e/ou estimularem atividades de lazer que promovam o desenvolvimento saudável da pessoa (Sarriera, Paradiso et al., 2007).

A hipótese de associação entre lazer e condutas desviantes foi estudada por Formiga (2009), que encontrou que 
hábitos de lazer hedonistas (com características egoístas e individualistas) associaram-se positivamente a condutas desviantes em jovens. Por outro lado, observou-se que os hábitos de lazer instrutivos e lúdicos associaram-se negativamente a condutas desviantes. $\mathrm{O}$ autor defende que, ao estimular hábitos de lazer instrutivos e lúdicos, a família e a escola podem atuar como fatores de proteção a condutas desviantes, ao organizar e administrar os espaços de diversão do jovem, com o objetivo de manter os jovens e seu entorno social saudáveis.

Outra abordagem ao estudo do lazer é a econômica, em que o lazer seria definido como o oposto do trabalho profissional, como o momento do não-trabalho. Esses estudos têm foco no uso do tempo entre os vários papéis que as pessoas assumem, no grau de organização das pessoas no uso do tempo liberado do trabalho, nas variáveis pessoais e contextuais associadas aos diferentes tipos de uso do tempo e outras questões mais específicas. Sob essa abordagem, há maior preocupação em revelar como essas práticas se caracterizam, e não em tentar responder por que as atividades de lazer se constituem de maneiras específicas ou as motivações das pessoas ao se envolver com essas ações (Ateca-Amestoy, Serrano-del-Rosal, \& Vera-Toscano, 2008; Becker, 1965; Hurst, 2008).

Por sua vez, a perspectiva sociológica aborda o lazer como o "tempo orientado para a realização da pessoa com fim último" (Dumazedier, 1974, p. 91). A pessoa consegue aproveitar esse tempo quando se libera das obrigações de trabalho, familiares, sócio-espirituais e sócio-políticas. Desse modo, o lazer não é compreendido como sinônimo de tempo livre, uma vez que este é limitado pelo tempo de trabalho profissional e outras atividades improdutivas, ainda que ligadas à produção (higiene do local de trabalho, recolhimento de ferramentas, etc), do tempo de deslocamento entre o trabalho e a casa, e do tempo destinado às obrigações domésticas ou familiares (tarefas ligadas à casa, educação de crianças, manutenção vital - sono, alimentação, higiene pessoal), das obrigações sócio-políticas e sócio-espirituais. Ainda sob essa concepção, o lazer pode ser definido como um conjunto mais ou menos estruturado de atividades com respeito às necessidades do corpo e do espírito: lazeres físicos, práticos, intelectuais, artísticos e sociais, dentro dos limites do condicionamento econômico, social, político e cultural de cada sociedade.

Também sobre a definição desse domínio, quatro propriedades são indicadas, a saber, o caráter libertário, desinteressado, hedonístico e pessoal. O caráter libertário envolve a compreensão de que o lazer resulta de uma livre escolha, sendo a liberação de obrigações institucionais, profissionais, familiares, sócio-políticas e sócio-espirituais. Já o caráter desinteressado destaca que o lazer não está fundamentalmente submetido a nenhum fim lucrativo ou utilitário. Por sua vez, o caráter hedonístico revela a busca de um estado de satisfação, tomado como um fim em si mesmo. Por fim, no que tange ao caráter pessoal, destaca-se que as funções expressas pelas atividades de lazer respondem às necessidades do indivíduo, face às obrigações primárias impostas pela sociedade.

O lazer está ligado à realização das virtudes desinteressadas do homem, concebido como um fim em si, em relação ou em contradição com as necessidades da sociedade. Em complemento, diz-se que o lazer possui três funções principais: divertimento, descanso e desenvolvimento pessoal (Dumazedier, 1974).

As quatro propriedades destacadas por Dumazedier (1974) aproximam-se do conceito de experiências autotélicas estudadas na teoria do fluxo. Csikszentmihalyi (1990, 1998) indica que fluxo é um estado no qual as pessoas estão tão envolvidas no que fazem que nada mais parece importar, uma vez que a experiência em si é tão prazerosa que mesmo quando há um grande prejuízo, ela é feita pelo simples motivo de fazê-la.

Nas situações de fluxo, as experiências tornam-se autotélicas, ou seja, vale a pena fazer a atividade por ela mesma (não há uma motivação externa ou objetivo utilitário). A motivação intrínseca tende a melhorar a sensação de bemestar e aumentar as emoções positivas, como um resultado de experiências desafiadoras. Considerando a teoria do fluxo e relacionando-a com os aportes sobre o lazer, as experiências de lazer, quando tornam-se experiências de fluxo, podem estimular o bem-estar por serem situações em que a pessoa faz algo em que há alta concentração/absorção e que a própria realização da tarefa motiva a execução da mesma (Rhoden, 2009).

Puig e Trilla (2004) conceituam o "ócio" de forma análoga à definição sociológica de lazer. Indicam que o ócio ocorre quando o homem, durante seu tempo livre (em oposição ao tempo que dedicamos ao trabalho), decide e gerencia livremente suas atividades, obtém prazer e satisfaz necessidades pessoais. Ao se remeter à origem etimológica da palavra "ócio", os autores retomam o conceito de que o ócio não consiste em não fazer nada, mas em realizar atividades de maneira não utilitária, que terão como resultado de mais longo prazo a formação não utilitária da pessoa. Assim, a razão do ócio está nele mesmo, não havendo objetivo maior em realizá-lo. Desse modo, lazer e ócio foram usados como sinônimos neste texto.

Mais recentemente, vários estudos vêm sendo desenvolvidos, dando seguimento às investigações da relação entre esse domínio e trabalho na sociedade de consumo (Albornoz, 2008, 2009; Aquino \& Martins, 2007; De Masi, 2000). Uma das fortes influências nesse campo é De Masi (2000), que pontua que o trabalho invade o tempo livre e priva as pessoas "das alegrias relacionadas ao tempo livre, a organização do lar, os afetos, a paternidade” (p. 177).

$\mathrm{O}$ autor defende que as máquinas devem fazer o trabalho repetitivo e com objetivo de máxima produção, mas que ao homem cabe o "ócio criativo", ou seja, a capacidade de pensar sobre, de refletir e de criar. Sugere que deve existir um equilíbrio entre as atividades de trabalho, estudo e lazer, defendendo a valorização e enriquecimento do tempo livre, por meio de uma redução da jornada de trabalho e pela possibilidade de dedicar-se à atividade intelectual e criativa. Nessa proposta, o ócio criativo permite que o homem se desenvolva como um todo, e que não seja definido exclusivamente pelo trabalho.

Em complemento a essas ideias, outros autores argumentam que muito frequentemente o lazer envolve atividades comerciais e produtivas, utilitárias, não 
possibilitando o desenvolvimento humano e as oportunidades de conhecer seus potenciais.

Nessa perspectiva, o lazer, paradoxalmente, pode contribuir para manter o status quo da sociedade, reforçando estereótipos e condutas alienantes e consumistas. Apesar da aparência utópica desses argumentos, defende-se que deve haver uma busca pela felicidade por meio das oportunidades de desenvolvimento, que devem ser descobertas por cada pessoa na arte, na cultura, em não fazer nada, etc (Albornoz, 2010; Elizalde, 2011; Pinheiro \& Soares, 2009; Pinheiro, Rhoden \& Martins, 2010).

Assim, compreende-se que o ócio deve ser estimulado por três motivos principais, quais sejam, como melhora da situação ou condição de uma pessoa ou coletivo, como prevenção de doenças ou manutenção da saúde e como uma experiência psicológica satisfatória.

O impacto positivo do ócio sobre a saúde física é o principal argumento usado para promover a prática esportiva e do ócio entre a população. No entanto, essa visão de que o ócio promove apenas benefícios físicos é reducionista, considerando a gama de variáveis em questão e a complexidade das mesmas (benefícios nas áreas psicológica, social, etc).

Outro tipo de benefício menos visível do ócio incide sobre os aspectos pessoais ou sociais, no sentido de maior percepção de bem-estar e qualidade de vida. A prática do ócio, a longo prazo, permitiria o desenvolvimento de habilidades pessoais e sociais que podem promover a busca por benefícios mais amplos, ou seja, as metas que as pessoas querem buscar ao longo de toda sua existência (Monteagudo, 2008).

Desse modo, o estudo do lazer ganha relevância ao se considerar várias instâncias, a saber, pessoal, familiar, societal, empresarial e governamental. Pesquisas sobre a temática podem facilitar o planejamento de atividades de lazer, tanto com caráter preventivo como terapêutico.

Em uma revisão de publicações sobre lazer entre 1891 e 2006, observou-se grande crescimento da produção na área, especialmente a partir de meados da década de 1990. Destacou-se a grande variedade de áreas do conhecimento que se ocupam desta temática, com maior força dos estudos da Sociologia e, mais recentemente, da Educação Física, incluindo também estudos da Psicologia. Complementarmente, boa parte dos textos publicados abordou os usos do tempo livre, descrevendo como a população organiza o seu cotidiano e aproveita os momentos de lazer.

Ainda, os estudos do lazer estiveram associados principalmente a dois eixos, quais sejam, o direito do trabalhador ao tempo liberado do trabalho e a necessidade de um uso "adequado" desse tempo, o que sugere a necessidade do governo atuar com políticas públicas para evitar o uso desse tempo de forma nociva e; numa outra perspectiva, a recreação como uma forma de complementar a formação cultural da população, indo em direção à formação de uma sociedade que atende principalmente a fins elitistas/burgueses (Peixoto, 2007).

Desse modo, mostra-se relevante o estudo do estado da arte do lazer no contexto das pesquisas científicas brasileiras. A presente pesquisa documental teve como objetivo analisar artigos científicos sobre a temática lazer e ócio publicadas em revistas científicas brasileiras, de modo a possibilitar a identificação de áreas já exploradas e de lacunas na produção do conhecimento.

\section{Método}

\section{Material e Procedimentos}

Foi realizada uma busca em 20 de Fevereiro de 2014, no site da Biblioteca Virtual da Saúde (BVS- www.bvs-psi. org.br), por textos completos, com os descritores "lazer" e "ócio", presentes no título dos artigos. Essa busca identificou 136 artigos, porém alguns deles não foram aproveitados: havia 14 artigos em duplicidade entre as bases do Pepsic e Scielo, outros 13 foram excluídos por se tratarem de artigos sobre o estudo de doenças em espaços de lazer ou da ruminação de certos animais (e não relacionados ao conceito de lazer propriamente dito) ou eram resumos de dissertações em andamento, de modo que não foi possível obter as informações mínimas necessárias para a análise; além da exclusão de uma resenha de livro. Desse modo, foram aproveitados 108 artigos para análise. Para a compreensão dos dados informados nos artigos, foram estabelecidas 13 categorias de análise, cujas definições vêm a seguir.

Ano de publicação: ano em que o artigo foi publicado.

Região geográfica: Estado brasileiro ao qual o primeiro autor do artigo encontrava-se vinculado e, no caso de autores que moravam fora do Brasil, o dado foi categorizado como pertencente a universidades ou empresas estrangeiras.

Categoria da Instituição de Ensino: descreve se a instituição à qual o primeiro autor estava vinculado é pública ou particular ou, no caso de autores estrangeiros, o dado foi categorizado como não informado, pois não foi possível localizar essa informação no artigo.

Modalidade de artigo: foram utilizados dois critérios, sendo o primeiro reflexão teórica, que abordou algum referencial teórico, a fim de compreender uma situação singular; e o segundo, relato de pesquisa, que envolveu tanto pesquisas com participantes como documentais.

Área do conhecimento: área do programa de PósGraduação ao qual o primeiro autor estava vinculado, relatado no mini-currículo presente no artigo ou, nos casos em que não havia essa informação, a mesma foi recuperada por meio da plataforma do Currículo Lattes. Adotou-se essa estratégia para definição da área do conhecimento da pesquisa pois pressupõe-se que os autores publicam seus artigos científicos de acordo com as linhas de pesquisa com as quais estão envolvidos no momento presente, ainda que tenham feito graduação ou doutorado em áreas diversas das que atuam no momento atual.

Palavras-chave/temática: consideraram-se as três primeiras palavras-chave que apareceram nos resumos, mesmo que os artigos apresentassem um número maior destas.

Quantidade de participantes: tamanho da amostra utilizada, no caso de relatos de pesquisa.

Idade dos participantes: categorizou-se a amostra de acordo com a faixa etária relatada, indo desde a infância 
até os idosos. Em alguns casos, a população era de uma faixa etária que incluía duas categorias (exemplo: crianças e adolescentes) e a informação foi incluída como população mista. Não foi possível trabalhar com a idade exata dos participantes, pois grande parte dos artigos não apresentou os dados sobre a amplitude, média e desvio-padrão da idade da amostra.

Modalidade dos participantes: referiu-se às características específicas que distinguem os participantes do estudo, tais como "membros da igreja", "atletas", "presidiários", e outros.

Procedimento de análise de dados: indicou-se o tipo de procedimento de análise adotado na pesquisa como qualitativos, quantitativos ou mistos.

Material de coleta de dados: referiu-se ao uso de questionários, entrevistas, observação, dois ou mais métodos usados em conjunto (exemplo: entrevista e questionário utilizados conjuntamente).

Revista de publicação: nome da revista em que o artigo foi publicado.

Abordagem de estudo do lazer: analisou-se qual dos aspectos do lazer foi abordado no artigo, sendo o artigo classificado em uma das quatro categorias: lazer em termos gerais (sentido dado ao lazer), tipo de atividades de lazer, atitudes frente o lazer e atividades físicas praticadas durante os momentos de lazer.

Como procedimentos de análise, serão apresentadas as frequências das categorias supracitadas e, em alguns casos, será feita a análise do Qui-quadrado para verificar diferenças de frequência ao comparar categorias distintas.

\section{Resultados}

Os artigos recuperados datam de 1997 a 2013, conforme Figura 1. Observa-se maior crescimento a partir de 2007, o que sugere um interesse mais recente no tema. Adicionalmente, verifica-se que desde 2009 há uma certa manutenção na produção sobre a temática.

As áreas do conhecimento que mais se destacaram no estudo da temática foram Educação Física $(f=39)$, Psicologia $(f=18)$, Epidemiologia $(f=9)$, Enfermagem ( $\mathrm{f}=7)$, Nutrição $(f$ $=7)$ e Educação/Pedagogia $(f=7)$. Sobre a região geográfica à qual o primeiro autor do artigo estava vinculado, os artigos foram gerados por pesquisadores de 14 diferentes Estados brasileiros, sendo a maior parte dos Estados de São Paulo (30,6\%), seguidos por Rio de Janeiro (13,9\%), Rio Grande do Sul (13\%) e Santa Catarina (7,4\%). A maioria dos autores estava vinculado a instituições públicas (estaduais ou federais - 76,9\%), havendo também uma participação de 7,4\% de pesquisadores de universidades estrangeiras. A maioria dos artigos foi de pesquisa (70,4\%), havendo 26 de cunho teórico, outros cinco eram revisões sistemáticas da literatura e um estudo de caso.

Sobre a quantidade de participantes das pesquisas em que essa análise era aplicável $(f=76)$, houve grande participação de pesquisas que se auto-intitularam epidemiológicas, que contaram com mais de 1000 participantes $(f=30)$, seguidas por pesquisas com 1 e 30 pessoas $(f=20), 101$ a $500(f=13)$, de 31 a $100(f=7)$, e de 501 a $1000(f=5)$. Uma pesquisa, de caráter qualitativo, não informou a quantidade de participantes. Desse modo, verificou-se uma tendência

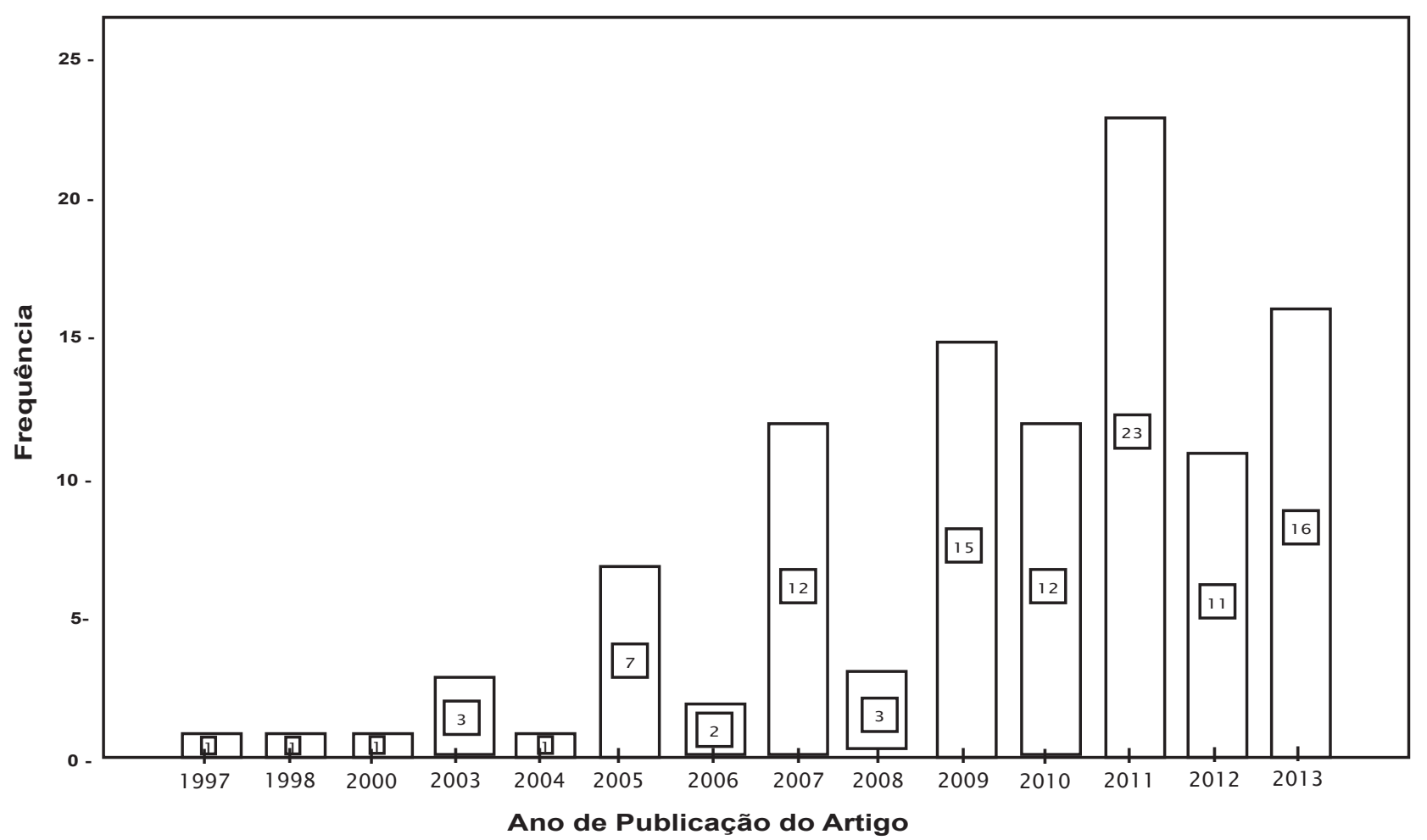

Figura 1. Quantidade de artigos publicados por ano 
Tabela 1. Descrição das amostras utilizadas nos artigos de pesquisa

\begin{tabular}{|c|c|c|}
\hline Amostra & Frequência & Percentual \\
\hline População geral com idades variadas & 19 & 25 \\
\hline Estudantes do Ensino Fundamental, Médio ou Universitário & 16 & 21 \\
\hline Trabalhadores & 15 & 20 \\
\hline Idosos & 8 & 11 \\
\hline Adolescentes de classe popular & 3 & 4 \\
\hline Portadores de doenças crônicas & 3 & 4 \\
\hline Mulheres no pós-parto & 2 & 3 \\
\hline Pessoas com baixo nível sócio-econômico & 2 & 3 \\
\hline Líderes comunitários & 1 & 1 \\
\hline Presidiários & 1 & 1 \\
\hline Membros da igreja-fiéis & 1 & 1 \\
\hline Usuários de drogas & 1 & 1 \\
\hline Pessoas hospitalizadas & 1 & 1 \\
\hline Mães de bebês em risco & 1 & 1 \\
\hline Residentes do curso de Medicina & 1 & 1 \\
\hline Adultos de comunidade ribeirinha & 1 & 1 \\
\hline Total & 76 & 100 \\
\hline
\end{tabular}

Tabela 2. Comparação entre área do conhecimento dos primeiros autores do artigo e abordagem do lazer.

\begin{tabular}{|c|c|c|c|c|c|}
\hline $\begin{array}{l}\text { Área do conhecimento / } \\
\text { Abordagem do lazer }\end{array}$ & $\begin{array}{c}\text { Tipos de } \\
\text { atividades de } \\
\text { lazer }\end{array}$ & $\begin{array}{l}\text { Atitudes sobre o } \\
\text { lazer }\end{array}$ & $\begin{array}{l}\text { Atividade física } \\
\text { durante tempo } \\
\text { de lazer }\end{array}$ & $\begin{array}{l}\text { Lazer em geral- } \\
\text { sentido do lazer }\end{array}$ & Total \\
\hline Educação Física & 11 & 0 & 22 & 6 & 39 \\
\hline Psicologia & 9 & 1 & 0 & 8 & 18 \\
\hline Epidemiologia & 2 & 0 & 7 & 0 & 9 \\
\hline Enfermagem & 4 & 0 & 3 & 0 & 7 \\
\hline Nutrição & 1 & 0 & 6 & 0 & 7 \\
\hline Educação/Pedagogia & 6 & 0 & 0 & 1 & 7 \\
\hline Medicina & 1 & 0 & 4 & 0 & 5 \\
\hline História & 2 & 0 & 0 & 1 & 3 \\
\hline Sociologia & 1 & 0 & 0 & 1 & 2 \\
\hline Administração & 1 & 0 & 0 & 1 & 2 \\
\hline Ciências Sociais & 0 & 0 & 1 & 1 & 2 \\
\hline Artes & 0 & 0 & 0 & 2 & 2 \\
\hline Filosofia & 0 & 0 & 0 & 2 & 2 \\
\hline Saúde Publica & 1 & 0 & 0 & 0 & 1 \\
\hline Antropologia & 1 & 0 & 0 & 0 & 1 \\
\hline Fonoaudiologia & 1 & 0 & 0 & 0 & 1 \\
\hline Total & 41 & 1 & 43 & 23 & 108 \\
\hline
\end{tabular}


à coleta de dados com amostras relativamente grandes ( $n$ $>100$ ), totalizando $64 \%$ dos casos válidos.

A maioria das pesquisas abordou exclusivamente adultos $(f=39), 13$ contaram com a participação de idosos, 12 com adolescentes, três avaliaram apenas crianças e nove abordaram pessoas de mais de uma faixa etária. Ao analisar características específicas da população, observou-se grande variedade, como se destaca na Tabela 1.

Apesar da variedade, é possível observar que as populações mais consultadas foram amostras da população geral $(f=19)$, em alguns casos baseadas em Censos, estudantes $(f=16)$, trabalhadores de variadas categorias profissionais $(f=15)$ e idosos $(f=8)$. É válido mencionar que os participantes foram incluídos na categoria que o estudo destacava. Então, apesar de adolescentes de classe popular também serem estudantes, essa não foi a característica destacada no estudo, de modo que a inclusão em categorias sempre foi feita de acordo com a característica da amostra que o artigo destacou como mais importante.

A maioria das pesquisas foi quantitativa $(68,4 \%)$, sendo $27,6 \%$ qualitativas e $3,9 \%$ mistas. A maioria das investigações usou questionários $(53,9 \%)$, seguidos por entrevistas $(22,4 \%)$, dois ou mais métodos de coleta de dados $(21,1 \%)$ e observações $(2,6 \%)$.

Sobre a abordagem de estudo do lazer, esse foi principalmente abordado no sentido restrito das atividades físicas realizadas durante os momentos de lazer $(39,8 \%)$ e pelos estudos que descreviam tipos de atividades de lazer (38,\%). Em menor frequência, houve aqueles que abordavam teórica ou empiricamente o conceito do lazer/ócio (21,3\%) e as atitudes frente o lazer $(0,9 \%)$. Os artigos que abordavam as atividades físicas feitas nos momentos de lazer geralmente exploravam a temática da inatividade/sedentarismo e sua relação com variáveis sócio-demográficas, do mesmo modo que o ocorrido nos artigos que abordavam tipos de atividades de lazer (exemplos: Costa, Mascarenhas \& Wiggers, 2011; Farah et al., 2013; Lima, Fermino, Oliveira, Añez, \& Reis, 2013). Por sua vez, os artigos sobre o conceito do lazer tratavam dos possíveis benefícios pessoais e sociais dessas práticas, a relação entre as políticas públicas, consumo, utilitarismo e práticas de lazer, entre outros (exemplos: Albarnoz, 2010; Elizalde, 2011; Pinheiro \& Soares, 2009; Pinheiro et al., 2010). Assim, os benefícios físicos oriundos do lazer foram mais abordados em artigos de pesquisa, enquanto os benefícios psicológicos e sociais estiveram mais destacados em artigos teóricos. A comparação entre a área do conhecimento e abordagem de estudo do lazer foi feita por meio da análise do Qui-quadrado, tendo-se observado que as diferenças foram significativas $(p=0,005)$. A comparação pode ser visualizada na Tabela 2 .

Os estudos de autores da Psicologia dividiram-se entre os tipos de atividades de lazer $(f=9)$ e a conceituação do lazer/ócio $(f=8)$, enquanto os da Educação Física focaram principalmente nas atividades físicas realizadas nos momentos de lazer $(f=22)$, do mesmo modo que os autores da Epidemiologia e Nutrição. Por sua vez, os estudos da área da Enfermagem estiveram divididos entre os tipos de atividade de lazer realizadas e as atividades físicas de lazer, enquanto a Educação/Pedagogia destacou-se nos estudos sobre os tipos de atividade de lazer. Assim, observa-se uma priorização de estudos sobre aspectos mais superficiais relacionados ao lazer, não havendo um interesse tão expressivo pelo desenvolvimento conceitual do lazer e ócio.

Sobre as revistas em que os artigos foram publicados, houve um total de 46 revistas distintas, sendo as mais frequentes Motriz ( $f=12$, sendo nove artigos de pesquisa), Revista Mal-Estar e Subjetividade $(f=10$, sendo nove artigos teóricos), Cadernos de Saúde Pública $(f=9$, todos de pesquisa), Revista Brasileira de Educação Física e Esporte ( $f$ $=8$, dividindo-se entre cinco pesquisas e três artigos teóricos) e Revista de Saúde Pública ( $f=7$, todas pesquisas). Nos demais casos, houve frequência menor ou igual a quatro publicações por revista.

Houve um total de 86 palavras-chave distintas estudadas nos artigos consultados, excluindo os unitermos "lazer" e "ócio" propriamente, totalizando 244 palavras-chave analisadas. As mais frequentes envolveram a realização de atividades físicas durante momentos de lazer $(f=39)$, a promoção de políticas públicas voltadas para a saúde por meio de atividades de lazer $(f=22)$, a relação entre lazer e trabalho $(f=15)$, as associações entre estilo de vida e a prática de atividades de lazer $(f=14)$, os esportes realizados nos momentos de lazer $(f=13)$, a relação entre a juventude e as práticas de lazer $(f=11)$, os hábitos de saúde e sua relação com as atividades de lazer $(f=8)$, as possibilidades de educação para o lazer como uma forma de transformação pessoal e social $(f=7)$, as características do lazer entre idosos $(f=7)$ e a relação entre comportamentos de risco à saúde e as atividades de lazer $(f=6)$. Nos demais casos, as temáticas tiveram frequência menor que seis. A comparação entre a primeira palavra-chave e a área de conhecimento, feita por meio da análise do Qui-quadrado, foi estatisticamente significativa $(p=0,001)$ e os dados são expostos na Tabela 3 .

É possível observar que a Educação Física ocupou-se principalmente das atividades físicas/motoras realizadas nos momentos de lazer e das políticas públicas relacionadas à prática de atividade física nos momentos de lazer. Ao seu turno, os pesquisadores dos cursos de Epidemiologia e Nutrição também abordaram de forma mais destacada as atividades motoras realizadas durante o lazer. Nos demais casos, incluindo o da Psicologia, houve maior variação quanto às palavras-chave utilizadas nos artigos.

\section{Discussão}

Os objetivos deste estudo envolveram a análise de artigos que abordaram a temática "lazer" ou "ócio", no sentido de identificar as áreas de produção de conhecimento mais desenvolvidas e de apontar lacunas existentes no momento atual. Pesquisas com esse caráter são especialmente úteis para o planejamento de novos estudos, no sentido de concentrar os investimentos em aspectos sub-explorados.

Este estudo revelou uma predominância de estudos de pesquisadores da área da Educação Física, Psicologia, Epidemiologia, Enfermagem e Nutrição, o que, de certa forma, é surpreendente, dado que em outro momento histórico, as pesquisas da Sociologia e da Economia eram dominantes (Dumazedier, 1974; Peixoto, 2007). Assim, isso aponta para uma nova configuração dos estudos do lazer, que, 
Tabela 3. Comparação entre palavras-chave e área do conhecimento de vinculação dos primeiros autores, incluindo apenas palavras-chave com frequência superior a 1

\begin{tabular}{|c|c|c|c|c|c|c|c|c|c|c|c|c|c|}
\hline $\begin{array}{c}\text { Área do } \\
\text { conhecimento } \\
\text { Palavras-chave }\end{array}$ & $\begin{array}{c}\text { Perfil do } \\
\text { consumidor }\end{array}$ & Saúde & $\begin{array}{l}\text { Atividade } \\
\text { motora }\end{array}$ & Esporte & $\begin{array}{c}\text { Estilo } \\
\text { de vida }\end{array}$ & Cultura & $\begin{array}{l}\text { Políticas } \\
\text { publicas }\end{array}$ & Trabalho & Idosos & Escala & Juventude & Consumo & Total \\
\hline Educação Física & 0 & 1 & 17 & 2 & 2 & 1 & 6 & 2 & 0 & 0 & 0 & 0 & 31 \\
\hline Psicologia & 0 & 2 & 0 & 0 & 0 & 2 & 0 & 1 & 0 & 2 & 2 & 2 & 11 \\
\hline Epidemiologia & 0 & 0 & 8 & 0 & 0 & 0 & 0 & 0 & 0 & 0 & 0 & 0 & 8 \\
\hline Nutrição & 0 & 0 & 6 & 0 & 0 & 0 & 0 & 0 & 0 & 0 & 0 & 0 & 6 \\
\hline Enfermagem & 0 & 2 & 3 & 0 & 0 & 0 & 0 & 0 & 0 & 0 & 0 & 0 & 5 \\
\hline Medicina & 0 & 0 & 1 & 1 & 0 & 0 & 0 & 0 & 1 & 0 & 2 & 0 & 5 \\
\hline Pedagogia & 0 & 0 & 0 & 1 & 0 & 0 & 1 & 0 & 1 & 0 & 2 & 0 & 5 \\
\hline História & 0 & 0 & 0 & 0 & 0 & 0 & 0 & 1 & 0 & 0 & 1 & 0 & 2 \\
\hline Administração & 1 & 0 & 0 & 0 & 0 & 0 & 0 & 1 & 0 & 0 & 0 & 0 & 2 \\
\hline Ciências Sociais & 0 & 0 & 0 & 1 & 0 & 0 & 0 & 0 & 0 & 0 & 0 & 0 & 1 \\
\hline Antropologia & 1 & 0 & 0 & 0 & 0 & 0 & 0 & 0 & 0 & 0 & 0 & 0 & 1 \\
\hline Artes & 0 & 0 & 0 & 0 & 0 & 1 & 0 & 0 & 0 & 0 & 0 & 0 & 1 \\
\hline Fonoaudiologia & 0 & 0 & 0 & 0 & 0 & 0 & 0 & 0 & 0 & 0 & 1 & 0 & 1 \\
\hline Total & 2 & 5 & 35 & 5 & 2 & 4 & 7 & 5 & 2 & 2 & 8 & 2 & 79 \\
\hline
\end{tabular}

atualmente, concentrou-se nos benefícios físicos gerados com as práticas do lazer. Apesar desse resultado, ainda não se observa uma interlocução entre áreas, não se observando investigações mais amplas e aprofundadas sobre o lazer. Uma aproximação já existente e que deveria se intensificar é entre a Sociologia e a Psicologia, de modo a compreender as motivações, possíveis riscos e benefícios e relações entre o momento sócio-histórico vivenciado com as práticas de lazer (Albarnoz, 2008, 2009, 2010; De Masi, 2000; Dumazedier, 1974; Formiga, 2009; Sarriera, Tatim et al., 2007; Sarriera, Paradiso et al., 2007).

Apesar de a maioria dos artigos serem relatos de pesquisas empíricas, houve também uma contribuição expressiva de estudos teóricos. Esse panorama é considerado favorável, uma vez que se entende que as formulações teóricas estão sendo empiricamente testadas, o que favorece a reelaboração de teorias mais adequadas às realidades estudadas.

Ao observar a variedade das características das populações estudadas, verifica-se que as pesquisas estão indo na direção de estudos de pessoas com características específicas (Alves, Montenegro, Oliveira, \& Alves, 2005); Azevedo \& Carvalho, 2006; Camargo \& Bueno, 2003, entre outros), o que permite o planejamento de intervenções mais ajustadas às características das populações-alvo. Assim, acredita-se que a sociedade terá mais benefícios com as pesquisas realizadas sobre o lazer, visto que, quando as intervenções forem colocadas em prática, haverá maior fundamentação sobre as necessidades de populações específicas.

No que tange a abordagem de estudo do lazer, esse foi principalmente analisado no sentido específico das atividades físicas realizadas durante os momentos de lazer e dos tipos de atividades de lazer. Sobre esse enfoque, há várias críticas de autores de áreas variadas sobre a existência de um estudo superficial sobre o lazer, que desconsidera aspectos subjetivos, as relações com a sociedade de consumo, a perpetuação de desigualdades por meio das práticas de lazer, entre outros (Albornoz, 2008, 2009, 2010; Elizalde, 2011; Pinheiro \& Soares, 2009; Pinheiro et al., 2010).

Os estudos de autores da Psicologia dividiram-se entre os tipos de atividades de lazer e o estudo do conceito do lazer, enquanto os da Educação Física focaram o lazer como uma atividade física, do mesmo modo que os autores da Epidemiologia e Nutrição. Por sua vez, os estudos da área da Enfermagem estiveram divididos entre os tipos de atividade de lazer realizadas e as atividades físicas de lazer. Assim, observou-se uma predominância de estudos sobre os aspectos físicos relacionados ao lazer, denotando uma carência de estudos que abordem os benefícios psicológicos e sociais relacionados às práticas de lazer, assim como que abordem os riscos associados a certos hábitos de lazer.

Mais detalhadamente, a Educação Física ocupou-se dos estilos de vida ou características sócio-demográficas relacionadas à prática de atividade física nos momentos de lazer ou ligadas ao sedentarismo e doenças crônicas (Kac et al., 2007; Martins, Assis, Nahas, Gauche, \& Moura, 2009; Pitanga \& Lessa, 2005, entre outros). Assim, essa área tem contribuído de forma expressiva no sentido de colaborar para a identificação de fatores que podem estimular a prática de atividades físicas nos momentos de lazer, que podem ser úteis especialmente para o planejamento das políticas públicas governamentais, porém que não contribuem para mudanças que possam surgir a partir de ações da própria comunidade. A prática de realizar estudos sobre o lazer como simples ocupação ativa do corpo no tempo do lazer é criticada por diversos autores, entre eles Bacheladenski e Mattielo Júnior (2010), que referem à importância de promover a saúde por meio do lazer de forma emancipatória e duradoura, e não de forma improvisada e esporádica.

A predominância de estudos de aspectos físicos relacionados ao lazer também se observou no estudo das palavras-chave dos artigos, em que a realização de atividades físicas durante momentos de lazer teve a maior frequência de citações. Contudo, por meio dessa análise percebeu-se que 
outras questões são exploradas, ainda que principalmente relacionadas aos aspectos físicos. Entre elas, encontram-se a da promoção de políticas públicas voltadas para a saúde por meio de atividades de lazer, o estudo dos hábitos de lazer dos jovens, a relação entre lazer e trabalho, a prática de esportes durante o tempo de lazer e as formas de melhorar a saúde por meio do lazer. A ampliação dos eixos de estudo poderia dar sustentação para a elaboração de propostas para o estímulo à participação da família, escola e sociedade na organização e administração das atividades de lazer (Formiga, 2009; Sarriera, Tatim et al., 2007; Sarriera, Paradiso et al., 2007), assim como do governo, no planejamento de políticas públicas que promovam hábitos saudáveis e que tentem evitar o desenvolvimento da violência (Monteagudo, 2008; Peixoto, 2007). De forma mais abrangente e sem ser passível de um "planejamento" por terceiros, a ampliação dos focos de estudo poderá favorecer a busca pelo ócio criativo, as experiências de flow, a descoberta dos potenciais pessoais desvinculada das práticas de consumo atreladas ao lazer, entre outros (Albarnoz, 2008, 2009, 2010; Aquino \& Martins, 2007; Csikszentmihalyi, 1990, 1998; De Masi, 2000; Dumazedier, 1974; Elizalde, 2011; Pinheiro \& Soares, 2009; Pinheiro et al., 2010; Rhoden, 2009).

\section{Considerações Finais}

Esta pesquisa revelou alguns dados não esperados, como o fato de pesquisadores da área da Sociologia, que anteriormente se destacavam no estudo do lazer (De Masi, 2000; Dumazedier, 1974; Peixoto, 2007), terem aparentemente parado de investir nos estudos da área, abrindo espaço para pesquisadores de outras áreas, como da Psicologia, Educação Física, Nutrição e Enfermagem. Apesar dessa queda na produção de artigos por pesquisadores da Sociologia, é possível que isso não expresse uma falta de investimento na temática, mas apenas que os pesquisadores podem estar priorizando a publicação em livros ou capítulos de livros, e não em artigos.

O incremento nas pesquisas a partir de 2007 é algo bastante favorável, porém verificou-se certa restrição no escopo dos estudos, no sentido de haver pesquisas focadas principalmente em resultados físicos obtidos por meio da prática do lazer. Assim, outros aspectos igualmente importantes têm sido deixados em segundo plano, como os benefícios psicológicos e sociais da prática do lazer, o desenvolvimento não utilitário da pessoa, o aumento na qualidade de vida e dos níveis de felicidade, e da ocorrência de experiências autotélicas durante as atividades de lazer (Csikszentmihalyi, 1990; De Masi, 2000; Dik \& Hansen, 2008; Dumazedier, 1974; Rhoden, 2009). Desse modo, sugere-se que os pesquisadores interessados na temática ampliem seu foco de estudo para aspectos menos "visíveis" no estudo do lazer, conforme defendido em outros contextos, fora do Brasil (Monteagudo, 2008).

De modo geral, acredita-se que a interlocução entre as diferentes áreas do conhecimento produzirá resultados bastante favoráveis para uma compreensão mais aprofundada sobre o assunto. Desse modo, defende-se que os benefícios físicos, psicológicos e sociais associados às práticas de lazer devem ser abordados em complementaridade. Acredita-se também que as possibilidades de desenvolvimento humano e realização pessoal por meio dessas atividades devem ser feitas considerando o momento histórico/cultural que vivemos, em que há uma constante invasão do trabalho nos espaços pessoais e que o tempo liberado das obrigações é cada vez mais escasso. Assim, conforme defendido por De Masi (2000), deve haver uma visão crítica sobre a relação entre trabalho e lazer, em que possa haver uma reflexão sobre o tema e uma busca pelo equilíbrio entre esses áreas. Por fim, destaca-se a importância da realização de estudos com populações em que haja uma participação ativa da família e comunidade nos hábitos de lazer, de modo a facilitar a compreensão dos fatores protetores às condutas desviantes realizadas nos momentos de lazer e um desinvestimento em práticas de lazer comerciais e alienantes.

Entre os limites do estudo, destaca-se que, pelo fato de se ter escolhido a Biblioteca Virtual em Saúde, artigos mais antigos, ainda não inseridos nessa base de dados, não foram contemplados. Contudo, considerando a abrangência das revistas incluídas no BVS, acredita-se que foi possível atingir o objetivo dessa pesquisa, no sentido de fazer um mapeamento do estado da arte dos estudos recentes sobre o lazer e ócio no Brasil. À guisa de conclusão, esta pesquisa revelou um interesse de novas áreas do conhecimento no estudo do lazer, o que deverá, a médio e longo prazo, ampliar o conhecimento sobre o domínio e apresentar novas contribuições para a sociedade.

\section{Referências}

Albornoz, S. G. (2008). Sobre o direito à preguiça de Paul Lafargue. Cadernos de Psicologia Social do Trabalho, 11(1), 1-17.

Albornoz, S. G. (2009). Jogo e trabalho: do homo ludens, de Johann Huizinga, ao ócio criativo, de Domenico De Masi. Cadernos de Psicologia Social do Trabalho, 12(1), 75-92.

Albornoz, S. G. (2010). Tempo livre e humanização: dúvidas e esperanças ante as novas possibilidades de lazer. Cadernos de Psicologia Social do Trabalho, 13(1), 89-101.

Alves, J. G. B., Montenegro, F. M. U., Oliveira, F. A., \& Alves, R. V. (2005). Prática de esportes durante a adolescência e atividade física de lazer na vida adulta. Revista Brasileira de Medicina do Esporte, 11(5), 291-294. doi:10.1590/S151786922005000500009

Aquino, C. A. B., \& Martins, J. C. O. (2007). Ócio, lazer e tempo livre na sociedade do consumo e do trabalho. Revista Mal-estar e Subjetividade, VII(2), 479-500.

Armstrong, P. I., \& Rounds, J. (2008). Linking Leisure Interests to the RIASEC World of Work Map. Journal of Career Development, 35(1), 5-22.

Ateca-Amestoy, V., Serrano-del-Rosal, R., \& Vera-Toscano, E. (2008). The leisure experience. The Journal of SocioEconomics, 37, 64-78.

Azevedo, R. P. C., \& Carvalho, A. M. A. (2006). O lugar da família na rede social do lazer após a aposentadoria. Revista Brasileira Crescimento e Desenvolvimento Humano, 16(3), 76-82.

Bacheladenski, M. S., \& Matiello Júnior, E. (2010). Contribuições do campo crítico do lazer para a promoção da saúde. Ciência e Saúde Coletiva, 15(2), 2569-2579. 
Becker, G. (1965). A theory of the allocation of time. Economic Journal, 75, 493-517.

Camargo, R. A. A., \& Bueno, S. M. V. (2003). Lazer, a vida além do trabalho para uma equipe de futebol entre trabalhadores de hospital. Revista Latino-Americana de Enfermagem, 11(4), 490-498.

Carvalho, V. A., \& Carlini-Cotrim, B. (1992). Atividades extracurriculares e prevenção ao abuso de drogas: uma questão polêmica. Revista de Saúde Pública, 26(3), 145-149.

Costa, J. M. D., Mascarenhas, F., \& Wiggers, I. D. (2011). O lazer eclipsado: registros sobre o programa "Escola Aberta". Motriz, 17(4), 569-578.

Csikszentmihalyi, M. (1990). Flow: The Psychology of Optimal Experience. New York: Harper and Row.

Csikszentmihalyi, M. (1998). Finding Flow: The Psychology of Engagement With Everyday Life. New York: Basic Books.

De Masi, D. (2000). O ócio criativo. Rio de Janeiro: Sextante.

Dik, B. J., \& Hansen, J. I. C. (2008). Following passionate interests to well-being. Journal of Career Assessment, 16(1), 86.

Dumazedier, J. (1974). Sociologia empírica do lazer. São Paulo: Perspectiva: SESC.

Elizalde, R. (2011). Ocio Transformacional y Contrahegemónico: "Disoñando" un Mundo Sustentable. Revista Mal-estar e subjetividade, XI(4), 1313-1336.

Gaudron, J. P., \& Vautier, S. (2007). Analyzing individual differences in vocational, leisure and family interests: a multitrait multi-method approach. Journal of Vocational Behavior, 70, 561-573.

Farah, B. Q., Barros, M. V. G., Farias-Júnior, J. C., Ritti-Dias, R. M., Barbosa, J. P. A. S., Lima, R. A., \& Nahas, M. V. (2013). Percepção de estresse: associação com a prática de atividades físicas no lazer e comportamentos sedentários em trabalhadores da indústria. Revista Brasileira Educação Física e Esporte, 27(2), 225-234.

Formiga, N. S. (2009). Hábitos de lazer e condutas desviantes: testagem de um modelo teórico em jovens. Boletim Academia Paulista de Psicologia, 30(79), 394-414.

Hur, Y-M, McGue, M., \& Iacono, W. G. (1996). Genetic and shared environmental influences on leisure-time interests in male adolescents. Personality and Individual Differences, 21(5), 791-801.

Hurst, M. (2008). Who participates in active leisure? Statistics Canada, pp. 27-33.

Kac, G., Resende, A. H. D., Pacheco, N., Araújo, D. M. R., Rocha, C. M. M. D., Sousa, E. B., . . Muniz, B. M. (2007). Fatores associados à prática de atividade física no lazer em mulheres no pós-parto. Revista Brasileira de Medicina do Esporte, 13(2), 86-90.
Lima, A. V., Fermino, R. C., Oliveira, M. P., Añez, C. R. R., \& Reis, R. S. (2013). Distância percebida até as instalações de lazer e sua associação com a prática de atividade física e de exercícios em adolescentes de Curitiba, Paraná, Brasil. Cadernos de Saúde Pública, 29(8), 1507-1521. doi:10.1590/ S0102-311X2013001200004

Martins, T. G., Assis, M. A. A., Nahas, M. V., Gauche, H., \& Moura, E. C. (2009). Inatividade física no lazer de adultos e fatores associados. Revista Saúde Pública, 43(5), 814-824.

Monteagudo, M. J. (2008). Consecuciones satisfactorias de la experiencia psicológica del ocio. Revista Mal-estar e Subjetividade, $\operatorname{VIII}(2), 307-325$.

Peixoto, E. (2007). Levantamento do estado da arte nos estudos de lazer: (Brasil) séculos XX e XXI - alguns apontamentos. Educação e Sociedade, 28(99), 561-586.

Pinheiro, K. F., Rhoden, I., \& Martins, J. C. O. (2010). A experiência do ócio na sociedade hipermoderna. Revista Mal-estar e subjetividade, X(4), 1131-1146.

Pinheiro, K. F., \& Soares, J. C. (2009). Cidade do lazer: expectativa de prazer. Revista Mal-estar e subjetividade, IX(3), 963-982.

Pitanga, F. J. G., \& Lessa, I. (2005). Prevalência e fatores associados ao sedentarismo no lazer em adultos. Cadernos de Saúde Pública, 21(3), 870-877.

Pratta, E. M. M., \& Santos, M. A. (2007). Lazer e uso de substâncias psicoativas na adolescência: possíveis relações. Psicologia: Teoria e Pesquisa, 23(1), 43-52.

Puig, J. M., \& Trilla, J. (2004). A pedagogia do ócio (2a ed.). Porto Alegre: Artmed.

Rhoden, I. (2009). O ócio como experiência subjetiva: contribuições da psicologia do ócio. Revista Mal-estar e Subjetividade, IX(4), 1233-1250.

Salles, L. M. F. (2010). A legitimação da violência nos espaços de lazer e na rua. Revista Mal-estar e Subjetividade, X(1), 211-232.

Sarriera, J. C., Paradiso, A. C., Mousquer, P. N., Marques, L. F., Hermel, J. S., \& Coelho, R. P. S. (2007). Significado do tempo livre para adolescentes de classe popular. Psicologia Ciência e Profissão, 27(4), 718-729.

Sarriera, J. C., Tatim, D. C., Coelho, R. P. S., \& Bucker, J. (2007). Uso do Tempo Livre por Adolescentes de Classe Popular. Psicologia Reflexão e Crítica, 20(3), 361-367.

Silva, J. M. A. P., \& Salles, L. M. F. (2010). Jovens, violência e escola: um desafio contemporâneo. São Paulo: Editora Unesp; São Paulo: Cultura Acadêmica.

Witt, P. A., \& Bishop, D. W. (2009). Situational antecedents to leisure behavior. Journal of Leisure Research, 41(3), 337-350.

Recebido em 15.11.2010

Primeira decisão editorial em 14.03.2013

Versão final em 07.03.2014

Aceito em 21.03.2014 